\title{
Isospin-rich nuclei in neutron star matter
}

\author{
Tapas Sil, ${ }^{1}$ J. N. De, ${ }^{2}$ S. K. Samaddar, ${ }^{1}$ X. Vinas, ${ }^{3}$ M. Centelles,${ }^{3}$ B. K. Agrawal, ${ }^{1,4}$ and S. K. Patra ${ }^{5}$ \\ ${ }^{1}$ Saha Institute of Nuclear Physics, 1/AF Bidhannagar, Kolkata 700064 , India \\ ${ }^{2}$ Variable Energy Cyclotron Centre, 1/AF Bidhannagar, Kolkata 700 064, India \\ ${ }^{3}$ Departament ECM, Facultat de Física, Universitat de Barcelona, Diagonal 647, E-08028 Barcelona, Spain \\ ${ }^{4}$ The Cyclotron Institute, Texas A\&M University, College Station, Texas 77843 \\ ${ }^{5}$ Institute of Physics, Sachivalaya Marg, Bhubaneswar 751 005, India
}

(Received 19 June 2002; published 29 October 2002)

\begin{abstract}
Stability of nuclei beyond the drip lines in the presence of an enveloping gas of nucleons and electrons, as prevailing in the inner crust of a neutron star, is studied in the temperature-dependent Thomas-Fermi framework. A limiting asymmetry in the isospin space beyond which nuclei cannot exist emerges from the calculations. The ambient conditions such as temperature, baryon density, and neutrino concentration under which these exotic nuclear systems can be formed are studied in some detail.
\end{abstract}

DOI: 10.1103/PhysRevC.66.045803

\section{INTRODUCTION}

In the outer region of the core of a neutron star, at densities $\sim 1.5 \times 10^{14} \mathrm{~g} / \mathrm{cm}^{3}$ or more, nucleons are distributed uniformly forming a homogeneous system [1-4]. At lower densities, in the inner crust of the star, inhomogeneities appear and then the positive charges get concentrated in individual nuclei of charge $Z$ which are embedded in a sea of neutrons, electrons, and possibly neutrinos, the whole system being charge neutral. The nuclei arrange themselves in a periodic lattice [5] due to the electrostatic interaction. In the typical conditions prevailing in the neutron star interior, the nuclei are in complete thermodynamic equilibrium with the environment and are assumed to be in $\beta$ equilibrium. They may well be beyond the neutron drip line $[1,6]$ known for laboratory nuclei with $(N-Z) / A \leqslant 0.35$. The excess neutrons of the very neutron rich nuclei present in the inner neutron star crust, which would otherwise decay under terrestrial conditions, are held together in stable equilibrium by the pressure exerted by the surrounding neutron sea.

The properties of the isospin-rich nuclei may be quite different from those of the terrestrial nuclei. For example, with increasing density, the nuclei may pass through different exotic shapes, namely, from spheres to cylinders, slabs, cylindrical holes, and spherical bubbles $[7,8]$. The presence of nonspherical nuclei could affect significantly the pinning of vortices and neutrino emission from neutron stars [9]. The existence of these exotic-shaped nuclei is, however, model dependent $[3,10]$. The external gas surrounding the nuclei may also influence the density distribution of these nuclei and thus may modify their size [1].

The nuclear equation of state plays the pivotal role in determining the macroscopic properties of a neutron star, such as its mass, radius, and moment of inertia [11]. In this context, the presence of different nuclear species in the outer and inner crustal regions provides the starting point for the consideration of some important aspects, such as its superfluid and elastic properties. However, for nuclei immersed in a nucleonic gas there are two basic concerns from a microscopic viewpoint. One, a thermodynamically consistent treatment of the coexistence of the two phases of nuclear matter (namely, the nuclear liquid and the surrounding gas) [12-
14], the other, a plausible description of the interface between the liquid and the gas [15-17]. It has been shown that this problem can be taken care of by solving the density profile in the subtraction procedure of Bonche, Levit, and Vautherin (BLV) $[18,19]$. In this method, the density profile of the liquid-plus-gas system and that of the gas are solved in a self-consistent procedure and then the extensive observables referring to the liquid are obtained as the difference between those of the two solutions. The influence of the surrounding gas on the surface energy is then automatically taken into account. The same concept can be applied to situations at zero temperature where drip nucleons occur and the system coexists with an outer nucleonic phase, as shown for very asymmetric cold semi-infinite nuclear matter in Ref. [17].

In this paper we focus on the effect of the external gas on the structure and stability of finite nuclei with large neutron excess, to have a broader understanding of the conditions under which these nuclei may exist in neutron star matter. A preliminary study for isolated nuclei immersed in a nucleonic gas at zero temperature has been done recently [20]; in the present work we extend these ideas in the appropriate astrophysical context. We also extend the calculations to nonzero temperature which is relevant at the formation stage of the neutron stars.

We assume that in the nuclear matter at subnuclear density the nuclei are located in a lattice. In order to simplify our calculation, the Wigner-Seitz approximation [5] is applied, where each lattice volume is replaced by a spherical cell of radius $R_{c}$, the nucleus being located at its center. The matter in each cell is taken to be charge neutral, i.e., the number of electrons is equal to the number of protons in the cell. The neutrino density in this cell is determined from the $\beta$-equilibrium condition. For a given average density of nuclear matter with a certain proton concentration, the density distribution is solved self-consistently. The calculations are performed in the finite temperature Thomas-Fermi formalism. The matter in the cell in which the protons and neutrons coexist does not define the nucleus itself, the nucleus is identified after subtraction of the gas part generated self-consistently, as in previous investigations of excited nuclei or asymmetric semi-infinite matter [17-19].

In Sec. II, the model employed in the calculations is in- 
troduced. The results and discussions are presented in Sec. III. Section IV contains the concluding remarks.

\section{MODEL}

In the inner region of the crust of a neutron star, we consider a mixture of neutrons, protons (some of which may exist as bound nuclear clusters), electrons, and neutrinos in thermodynamic equilibrium. We contemplate both cold matter as well as matter at a finite temperature. We ignore the contributions from alpha particles and also from photons. We further ignore the plasma effects. All these effects are known to be rather small [21]. The nuclear clusters are assumed to be arranged in a body-centered cubic lattice which we approximate by Wigner-Seitz (WS) cells defined as spheres with radius $R_{c}$. Each cell is assumed to be electrically neutral and interactions among the cells are neglected. At the densities that we are interested in, the Fermi momenta of the electrons are much larger than their rest mass, the electrons are then extremely relativistic and can be assumed to be uniformly distributed in the cell. We assume the matter to be in $\beta$ equilibrium, i.e., the chemical potentials of the considered species fulfil

$$
\mu_{n}=\mu_{p}+\mu_{e}-\mu_{\nu}
$$

At a fixed temperature, under the condition of charge neutrality, the variables in the problem are the average baryon density $\langle\rho\rangle$, the proton fraction $Y_{p}=Y_{e}$ (the electron fraction), the neutrino fraction $Y_{\nu}$, and the lattice radius $R_{c}$. With the $\beta$-equilibrium condition given by Eq. (1), if $\mu_{n}$, $\mu_{p}$, and $\mu_{e}$ are known, then $\mu_{\nu}$ can be determined which in turn yields $\rho_{\nu}$ when the neutrinos are taken to be a degenerate Fermi gas. Then only three of the variables are left independent. The condition of $\beta$ equilibrium of the baryons with the electrons and neutrinos guarantees that the system has the minimum free energy. If one imposes further the constraint that the lattice sites contain a particular nuclear species with a given charge $Z_{c l}$ and baryon number $A_{c l}$ (immersed in a nucleonic gas or not), then there is only one independent variable.

To obtain the thermodynamic properties of the system of baryons, electrons, and neutrinos, we minimize the free energy of the system in the WS cell with the constraint of number conservation of the individual species. Under the conditions of charge neutrality and $\beta$ equilibrium, the relevant grand potential is given by

$$
\Omega=\mathcal{F}-\sum_{q} \mu_{q} A_{q}
$$

where $\mathcal{F}$ is the free energy and the index $q=(n, p)$ refers to neutrons and protons. The free energy has the following expression:

$$
\begin{aligned}
& \mathcal{F}\left(\langle\rho\rangle, Y_{p}, Y_{\nu}, T\right) \\
& \quad=\int\left[\mathcal{H}(r)-T s(r)+\mathcal{E}_{c}(r)+f_{e}\left(\rho_{e}\right)+f_{\nu}\left(\rho_{\nu}\right)\right] d \mathbf{r}
\end{aligned}
$$

In Eq. (3) the integration is over the volume of the WS cell. Here $\mathcal{H}$ refers to the baryonic energy density excluding the Coulomb energy, $s$ is the entropy density of the baryons, $\mathcal{E}_{c}$ the Coulomb energy density of the system, and $f_{\nu}, f_{e}$ are the free energy densities of the neutrinos and of the electrons (Coulomb interaction excluded).

For the nuclear energy density, we use the Skyrme energy density functional. It is written as

$$
\begin{aligned}
\mathcal{H}(r)= & \frac{\hbar^{2}}{2 m_{n}^{*}} \tau_{n}+\frac{\hbar^{2}}{2 m_{p}^{*}} \tau_{p}+\frac{1}{2} t_{0}\left[\left(1+\frac{x_{0}}{2}\right) \rho^{2}-\left(x_{0}+\frac{1}{2}\right)\left(\rho_{n}^{2}\right.\right. \\
& \left.\left.+\rho_{p}^{2}\right)\right]-\frac{1}{16}\left[t_{2}\left(1+\frac{x_{2}}{2}\right)-3 t_{1}\left(1+\frac{x_{1}}{2}\right)\right](\nabla \rho)^{2} \\
& -\frac{1}{16}\left[3 t_{1}\left(x_{1}+\frac{1}{2}\right)+t_{2}\left(x_{2}+\frac{1}{2}\right)\right]\left[\left(\nabla \rho_{n}\right)^{2}+\left(\nabla \rho_{p}\right)^{2}\right] \\
& +\frac{1}{12} t_{3} \rho^{\alpha}\left[\left(1+\frac{x_{3}}{2}\right) \rho^{2}-\left(x_{3}+\frac{1}{2}\right)\left(\rho_{n}^{2}+\rho_{p}^{2}\right)\right]
\end{aligned}
$$

where $\rho=\rho_{n}+\rho_{p}$ and the effective mass of the nucleons $m_{q}^{*}$ is defined through

$$
\begin{aligned}
\frac{m}{m_{q}^{*}(r)}= & 1+\frac{m}{2 \hbar^{2}}\left\{\left[t_{1}\left(1+\frac{x_{1}}{2}\right)+t_{2}\left(1+\frac{x_{2}}{2}\right)\right] \rho\right. \\
& \left.+\left[t_{2}\left(x_{2}+\frac{1}{2}\right)-t_{1}\left(x_{1}+\frac{1}{2}\right)\right] \rho_{q}\right\} .
\end{aligned}
$$

In the numerical calculations we shall employ the SKM* interaction, whose parameters can be found in Ref. [22]. In the Thomas-Fermi approximation the kinetic energy density $\tau_{q}$ is given by

$$
\begin{gathered}
\tau_{q}(r)=\frac{3}{5}\left(3 \pi^{2}\right)^{2 / 3} \rho_{q}^{5 / 3} \text { at } T=0, \\
\tau_{q}(r)=\frac{1}{2 \pi^{2}}\left(\frac{2 m_{q}^{*} T}{\hbar^{2}}\right)^{5 / 2} J_{3 / 2}\left(\eta_{q}\right) \quad \text { at } T \neq 0 .
\end{gathered}
$$

The fugacity $\eta_{q}$ is obtained as

$$
\eta_{q}(r)=\left[\mu_{q}-V_{q}(r)\right] / T,
$$

where $V_{q}$ is the single-particle potential experienced by nucleons (including the Coulomb part for the protons). The nucleonic density $\rho_{q}$ is related to $\eta_{q}$ by

$$
\rho_{q}(r)=\frac{1}{2 \pi^{2}}\left(\frac{2 m_{q}^{*} T}{\hbar^{2}}\right)^{3 / 2} J_{1 / 2}\left(\eta_{q}\right) .
$$

The functions $J_{k}\left(\eta_{q}\right)$ in Eqs. (7) and (9) are the standard Fermi integrals.

For the entropy density of the nucleons, one has

$$
s(r)=\sum_{q}\left[(5 / 3) J_{3 / 2}\left(\eta_{q}\right) / J_{1 / 2}\left(\eta_{q}\right)-\eta_{q}\right] \rho_{q} .
$$


The direct part $\mathcal{E}_{c}^{d}$ of the Coulomb energy density $\mathcal{E}_{c}$ of the charged particles is

$$
\mathcal{E}_{c}^{d}(r)=\frac{1}{2}\left[\rho_{p}(r)-\rho_{e}\right] \int\left[\rho_{p}\left(r^{\prime}\right)-\rho_{e}\right] \frac{e^{2}}{\left|\mathbf{r}-\mathbf{r}^{\prime}\right|} d \mathbf{r}^{\prime},
$$

while the exchange part is computed in the Slater approximation,

$$
\mathcal{E}_{c}^{e x}(r)=-\frac{3}{4}\left(\frac{3}{\pi}\right)^{1 / 3} e^{2}\left[\rho_{p}^{4 / 3}(r)+\rho_{e}^{4 / 3}\right] .
$$

For $f_{e}$ and $f_{\nu}$ we use the standard expressions [23]. For the minimization procedure, we take recourse to the finite temperature Thomas-Fermi approximation which yields Eq. (8). This equation is solved self-consistently leading to the density distributions of neutrons and protons in the spherical WS cell.

Under the conditions of interest here, the baryonic fluid in the WS cell can congregate into nuclei (we consider only the spherical shapes) located at the center of the cell and may be embedded in a low density gas of nucleons. Near the edge of the cell, for hot or for isospin rich systems beyond the drip line, the baryon density profile is found to be practically uniform, which is identified as the low-density gas. To separate the nucleus from the embedding low-density environment, we follow the BLV procedure which has been used earlier in the Hartee-Fock (HF) framework [18] as well as in a Thomas-Fermi (TF) scheme [19] for a hot nucleus in coexistence with the vapor surrounding it. The method is based on the existence of two solutions to the HF or TF equations, one corresponding to the liquid phase with the surrounding gas (LG) and the other corresponding to the gas $(G)$ alone. One may note that this separation procedure is not exact, it is model dependent; however, in absence of a better prescription we follow this method.

For an isolated hot nucleus in equilibrium with the gas surrounding it, in the absence of Coulomb forces, the densities $\rho_{L G}^{q}$ and the gas density $\rho_{G}^{q}$ can be obtained from the variational equations,

$$
\begin{gathered}
\frac{\delta \Omega_{L G}^{N}}{\delta \rho_{L G}^{q}}=0, \\
\frac{\delta \Omega_{G}^{N}}{\delta \rho_{G}^{q}}=0,
\end{gathered}
$$

where $\Omega_{L G}^{N}$ and $\Omega_{G}^{N}$ are the grand potentials of the respective systems with the same chemical potential. In our procedure, the solution for $\rho_{L}=\rho_{L G}-\rho_{G}$, which may be called the liquid profile, is independent of the box volume in which the calculation is done. In the presence of the Coulomb force, however, the Coulomb repulsion increases with the box size, driving the protons to the edge of the box and leading ultimately to a divergence problem. In order to work out a convergent prescription, Bonche et al. [18] calculated the Coulomb energy $E_{c}$ from the subtracted proton density $\rho_{L G}^{p}$
$-\rho_{G}^{p}$ and obtained the density profile from the variation of the subtracted grand potential $\bar{\Omega}=\Omega_{L G}^{N}-\Omega_{G}^{N}+E_{c}$ with respect to both $\rho_{L G}^{q}$ and $\rho_{G}^{q}$.

In the astrophysical context, the situation is, however, different due to the presence of electrons. The Coulomb energies for the LG and $G$ phases are

$$
\begin{aligned}
E_{L G}^{c}= & \frac{1}{2} \int\left[\rho_{L G}^{p}\left(r^{\prime}\right)-\rho_{e}\right] \frac{e^{2}}{\left|\mathbf{r}-\mathbf{r}^{\prime}\right|}\left[\rho_{L G}^{p}(r)-\rho_{e}\right] d \mathbf{r} d \mathbf{r}^{\prime}, \\
E_{G}^{c}= & \frac{1}{2} \int\left[\rho_{G}^{p}\left(r^{\prime}\right)-\rho_{e}\right] \frac{e^{2}}{\left|\mathbf{r}-\mathbf{r}^{\prime}\right|}\left[\rho_{G}^{p}(r)-\rho_{e}\right] d \mathbf{r} d \mathbf{r}^{\prime} \\
& +\int \rho_{L}^{p}\left(r^{\prime}\right) \frac{e^{2}}{\left|\mathbf{r}-\mathbf{r}^{\prime}\right|}\left[\rho_{G}^{p}(r)-\rho_{e}\right] d \mathbf{r} d \mathbf{r}^{\prime} .
\end{aligned}
$$

The direct part of the single-particle Coulomb potentials ( $\delta E_{L G}^{c} / \delta \rho_{L G}^{p}$ and $\delta E_{G}^{c} / \delta \rho_{G}^{p}$ ) for the LG and the $G$ solutions is the same. It is given by

$$
V_{c}^{d}(r)=\int\left[\rho_{L G}^{p}\left(r^{\prime}\right)-\rho_{e}\right] \frac{e^{2}}{\left|\mathbf{r}-\mathbf{r}^{\prime}\right|} d \mathbf{r}^{\prime} .
$$

Since the system is charge neutral, the divergence problem does not arise. The solutions to the density profiles can be directly obtained from the variations of the total (with Coulomb) grand potentials $\Omega_{L G}$ for the liquid-plus-gas phase and $\Omega_{G}$ for the gas phase with respect to $\rho_{L G}^{q}$ and $\rho_{G}^{q}$, respectively. The resulting coupled equations are

$$
\begin{gathered}
T \eta_{L G}^{q}(r)+V_{L G}^{q}+V_{L G}^{c}\left(\rho_{L G}^{p}, \rho_{e}\right)=\mu_{q}, \\
T \eta_{G}^{q}(r)+V_{G}^{q}+V_{G}^{c}\left(\rho_{L G}^{p}, \rho_{G}^{p}, \rho_{e}\right)=\mu_{q} .
\end{gathered}
$$

At zero temperature they reduce to

$$
\begin{aligned}
& \left(3 \pi^{2}\right)^{2 / 3} \frac{\hbar^{2}}{2 m_{q}^{*}}\left(\rho_{L G}^{q}\right)^{2 / 3}+V_{L G}^{q}+V_{L G}^{c}\left(\rho_{L G}^{p}, \rho_{e}\right)=\mu_{q}, \\
& \left(3 \pi^{2}\right)^{2 / 3} \frac{\hbar^{2}}{2 m_{q}^{*}}\left(\rho_{G}^{q}\right)^{2 / 3}+V_{G}^{q}+V_{G}^{c}\left(\rho_{L G}^{p}, \rho_{G}^{p}, \rho_{e}\right)=\mu_{q} .
\end{aligned}
$$

In Eqs. (18)-(21), $V_{L G}^{q}$ and $V_{G}^{q}$ refer to the nuclear part of the single-particle potential corresponding to the liquid-plusgas and the gas solutions. $V_{L G}^{c}\left(V_{G}^{c}\right)$ is the sum of the direct part of the Coulomb single-particle potential given by Eq. (17) and the exchange term $-e^{2}(3 / \pi)^{1 / 3}\left(\rho_{L G(G)}^{p}\right)^{1 / 3}$ for the protons in the LG $(G)$ phase. At zero temperature, if the cluster is within the drip lines, the cell does not contain any nucleonic gas. Equations (20) and (21) then automatically lead to a gas solution which is zero throughout.

The nucleonic chemical potential is determined from the constraint of nucleon number conservation. In the case of a given nuclear cluster with neutron number $N_{c l}$ and proton 
number $Z_{c l}$ embedded in a nucleonic gas, the conservation of the number of nucleons in the cluster requires [from Eqs. (18) and (19)]

$$
\begin{aligned}
\mu_{q}= & \frac{1}{A_{q}}\left\{\int\left[T \eta_{L G}^{q}(r)+V_{L G}^{q}(r)+V_{L G}^{c}(r)\right] \rho_{L G}^{q}(r) d \mathbf{r}\right. \\
& \left.-\int\left[T \eta_{G}^{q}(r)+V_{G}^{q}(r)+V_{G}^{c}(r)\right] \rho_{G}^{q}(r) d \mathbf{r}\right\} .
\end{aligned}
$$

Here $A_{q}$ refers to $N_{c l}$ or $Z_{c l}$. An equation similar to Eq. (22) follows from Eqs. (20) and (21) at zero temperature.

However, when $\langle\rho\rangle$ is given in a WS cell with a given proton concentration, the total number of neutrons $N$ and protons $Z$ in the cell is defined. Then, the chemical potential is obtained as

$$
\mu_{q}=\frac{1}{A_{q}} \int\left[T \eta_{L G}^{q}(r)+V_{L G}^{q}(r)+V_{L G}^{c}(r)\right] \rho_{L G}^{q}(r) d \mathbf{r},
$$

where now $A_{q}$ refers to $N$ or $Z$.

\section{RESULTS AND DISCUSSION}

In an earlier paper by some of the present authors [20], calculations at zero temperature on some structural properties of isolated nuclei much beyond the drip line were reported. In that case, the excess pressure exerted by the enveloping gas stabilizes the nucleus even beyond the nominal drip lines (which are defined by $\mu_{n}=0$ or $\mu_{p}=0$ in the Thomas-Fermi approach). We now place these calculations in a broader context related to the environment existing in the inner crust of a neutron star. We take cognizance of the presence of electrons and neutrinos existing in $\beta$ equilibrium with the neutrons and protons. We extend the calculations also at finite temperature. As mentioned earlier, the calculations are performed in the finite temperature Thomas-Fermi framework in a Wigner-Seitz lattice employing the SKM* interaction. For completeness, we also discuss the situation for the proton-rich nuclei, though they may not exist in the crustal matter of the neutron stars.

In the calculations on asymmetric infinite and semiinfinite nuclear matter in equilibrium with a drip phase, it was observed $[12,16,17]$ that the neutron-proton asymmetry could be increased arbitrarily till the two phases merge into a uniform system when the densities and the asymmetries of both phases become equal. On the contrary, in the selfconsistent TF calculation for isolated finite nuclei, it was found that one cannot add or remove neutrons from nuclei arbitrarily [20]. There exists a limiting neutron-proton asymmetry $I=\left(N_{c l}-Z_{c l}\right) / A_{c l}$ beyond which the system becomes thermodynamically unstable; establishing chemical equilibrium between the nuclear phase and the gas phase further becomes impossible.

In the realistic conditions considered in the present investigation, including the lattice effects, we calculate the stability limits at $T=0$ as shown in the upper panel of Fig. 1. We find that both the limiting asymmetry (full line) and the drip asymmetry (dashed line) are not very sensitive to the atomic

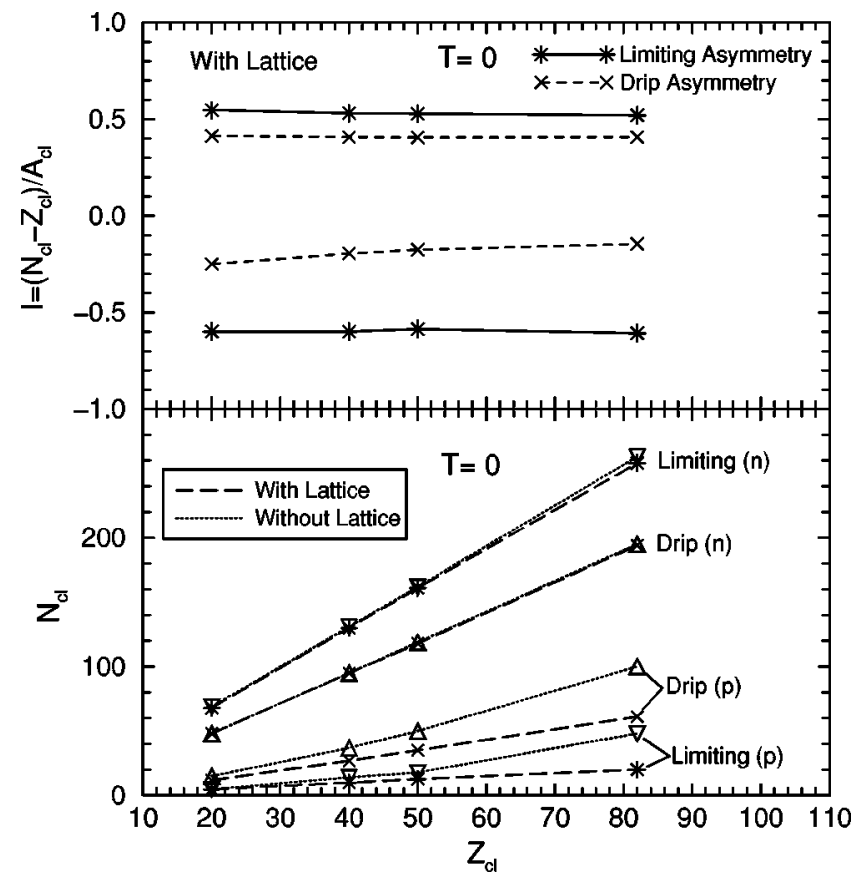

FIG. 1. The upper panel shows the drip line asymmetry and the limiting asymmetry as a function of the charge $Z_{c l}$ of the nuclear cluster in a lattice of size $R_{c}=15 \mathrm{fm}$ at $T=0$. The lower panel displays the same quantities in the $N_{c l}-Z_{c l}$ plane with and without inclusion of lattice effects.

number $Z_{c l}$ of the nucleus. The influence of the lattice electrons on both the drip lines and the limiting lines for neutrons and protons is manifested in the lower panel of Fig. 1. The neutron lines are nearly unaffected. The proton lines are, however, influenced significantly, particularly for the heavier clusters. Both the proton drip and limiting lines get extended with inclusion of the lattice effects because of the dilution of the Coulomb force in the presence of electrons. The calculations are done in a lattice size $R_{c}=15 \mathrm{fm}$. The results are found to be rather insensitive to the size of the lattice.

The influence of temperature on the drip and limiting lines, taking into account the lattice effects, is displayed in Fig. 2. There, we compare the results obtained at $T=0$ and at $T=6 \mathrm{MeV}$. Both the neutron and proton drip lines are extended at finite temperature as found earlier in calculations for isolated nuclei $[24,25]$. However, the neutron limiting line shrinks with temperature, whereas the proton limiting line remains essentially unaffected.

In Fig. 3 we present the density profile of neutrons (left panel) and protons (right panel) for an extremely neutronrich nucleus ${ }^{330} \mathrm{~Pb}$ (the neutron drip line is located at ${ }^{276} \mathrm{~Pb}$ ). We display calculations for both the case with lattice and the case without lattice (isolated nuclei) at $T=0$. At a finite temperature $T=6 \mathrm{MeV}$ we show the calculations only with lattice effects. The total density profile $\rho_{L G}^{q}$ of the liquid-plusgas phase is shown in the upper panels. For neutron-rich nuclei the influence of the electrons in the lattice is found to have only a nominal effect on the density distributions. The finite neutron density at the cell boundary even at $T=0$ reflects the presence of the neutron gas for a nucleus beyond the neutron drip line. With increasing temperature, the cen- 


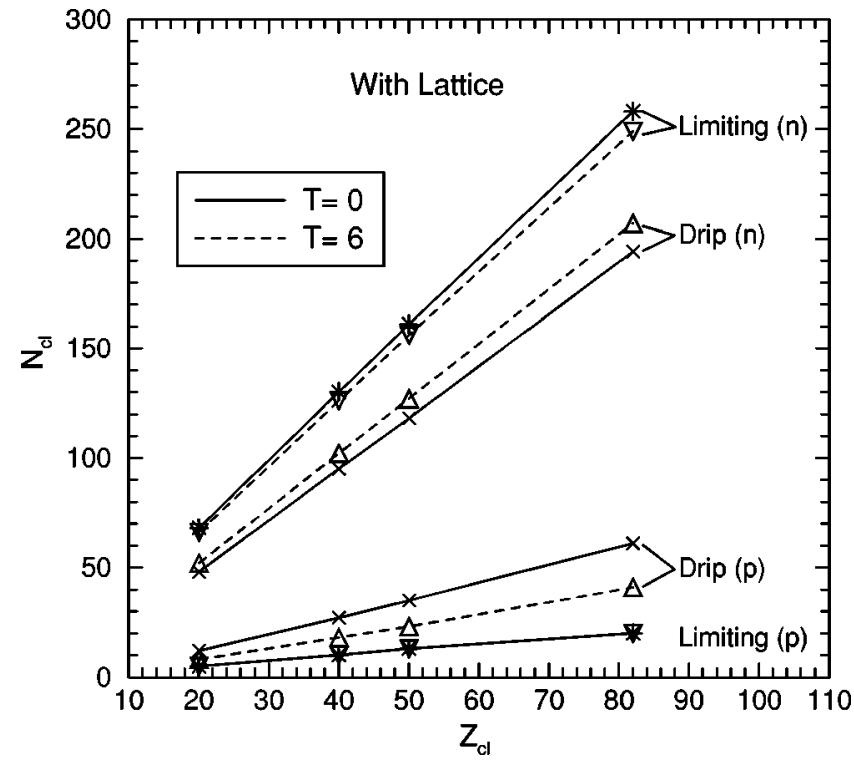

FIG. 2. The drip and the limiting asymmetries in the $N_{c l}-Z_{c l}$ plane at $T=0$ and $6 \mathrm{MeV}$ in a lattice of $R_{c}=15 \mathrm{fm}$. The limiting proton asymmetry coincides at both temperatures.

tral density is depleted with the appearance of a thickening tail. The gas densities $\rho_{G}^{q}$ are shown in the bottom panels and the liquid densities $\rho_{L}^{q}$ obtained as the difference between $\rho_{L G}^{q}$ and $\rho_{G}^{q}$ are shown in the central panels.

The density profile $\rho_{L}^{q}$ of the isolated cluster ${ }^{330} \mathrm{~Pb}$ is found to be independent of the size of the box. However, in a lattice, because of the modification of the Coulomb force in the presence of the electrons, the nucleonic densities in the cluster are box-size dependent, though weakly. The liquid density vanishes at a distance $r<R_{c}$ even at $T=6 \mathrm{MeV}$. The neutron gas density is found to be practically constant throughout the box; at finite temperature, the gas density is larger as expected. For this neutron-rich system, at $T=0$, the proton gas does not exist; however, at finite temperature ( $T$ $=6 \mathrm{MeV}$ ), a few protons are present in the gaseous state. In finite temperature calculations for isolated nuclei, the proton gas density profile is strongly polarized [18] due to the repulsion from the nuclear core. In the presence of the lattice electrons, the density polarization exists only in the vicinity of the core, at further distances the proton gas density is found to attain a nearly constant value.

The density distributions for an extremely proton-rich isotope ${ }^{140} \mathrm{~Pb}$ are shown in Fig. 4. The proton and neutron distributions at $T=0$ (both with and without lattice contributions) and at $T=6 \mathrm{MeV}$ with lattice effects are displayed in the left and right panels, respectively. The electrons in the lattice dilute the Coulomb repulsion thereby lowering the proton chemical potential, particularly for proton-rich heavy nuclei, as an effect of which the proton drip line is extended. For instance, for an isolated $\mathrm{Pb}$ nucleus the proton drip line is located at ${ }^{182} \mathrm{~Pb}$; the influence of the lattice electrons pushes it to ${ }^{143} \mathrm{~Pb}$. The total (LG) proton density distribution for the isolated ${ }^{140} \mathrm{~Pb}$ nucleus at zero temperature indicates the presence of a proton gaseous phase. This proton gas is strongly polarized as is clear from the proton gas density

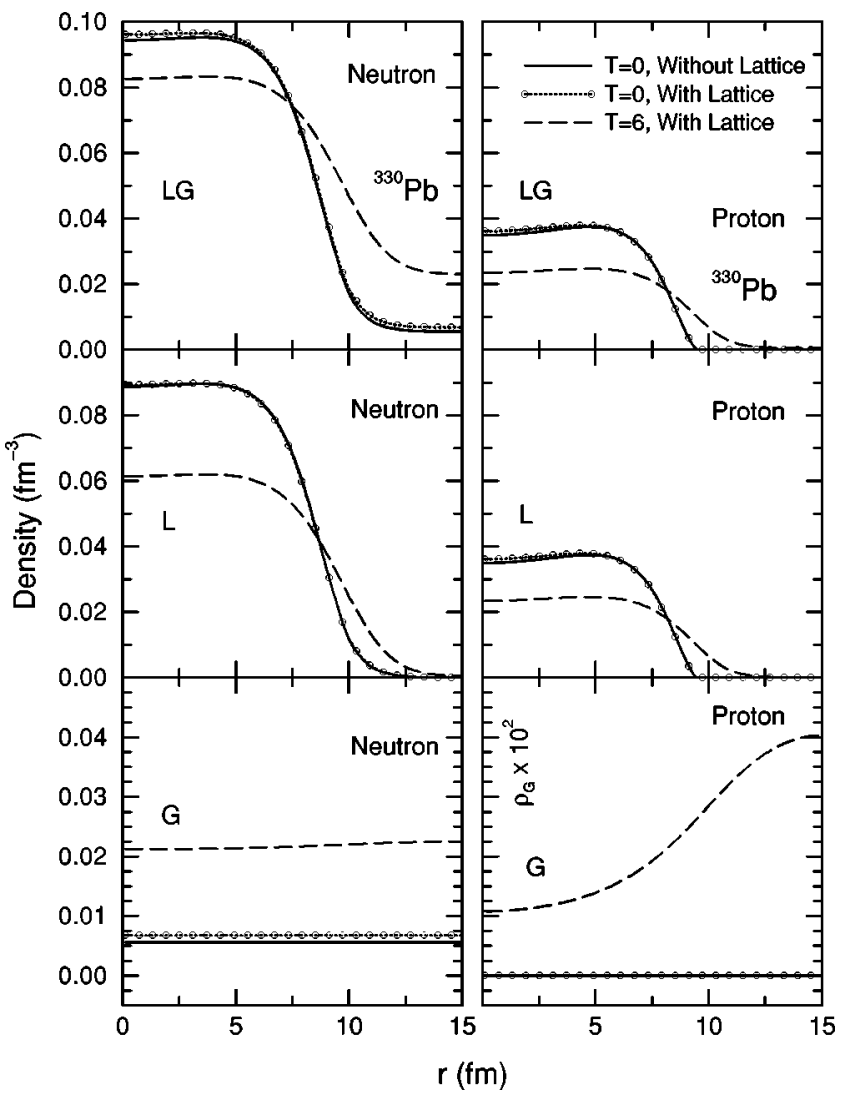

FIG. 3. The neutron (left panel) and proton (right panel) density profiles corresponding to the nuclear cluster ${ }^{330} \mathrm{~Pb}$ are shown with inclusion of lattice effects at $T=0 \mathrm{MeV}$ (open circles joined by dotted lines) and at $T=6 \mathrm{MeV}$ (dashed line). The full line corresponds to calculations for the isolated nucleus (without lattice) at $T=0$. The upper panels refer to the liquid-plus-gas density (LG) and the central panels correspond to the liquid density $(L)$. The lower panels show the gas density $(G)$.

distribution shown in the bottom panel. With inclusion of the lattice electrons, since the ${ }^{140} \mathrm{~Pb}$ nucleus is just beyond the modified drip line, the proton gas density is extremely dilute as is evident from the bottom left panel. The central panel of Fig. 4 indicates that the liquid density profiles with and without lattice corrections at $T=0$ are not much different. The influence of the lattice electrons on the neutron density distribution is negligible as displayed in the right panel. For the present system, there is no neutron gas at zero temperature. The characteristics of the density distributions at finite temperature are found to be basically the same as discussed in the context of a neutron-rich nucleus in Fig. 3.

All of the subsequent calculations we shall present are performed including the influence of the lattice electrons. In Fig. 5, the rms neutron and proton radii of the lead isotopes are displayed for $T=0$ as well as for $T=6 \mathrm{MeV}$. At zero temperature, except at the edges of limiting asymmetry, the rms radius changes almost linearly with the mass number. The faster change in radius near the edges points to the onset of instability. Similar observations were also noted in the calculations without lattice effects [20]. Temperature increases the rms radii, its effect being more pronounced near the stability limit. By comparing the upper and lower panels 


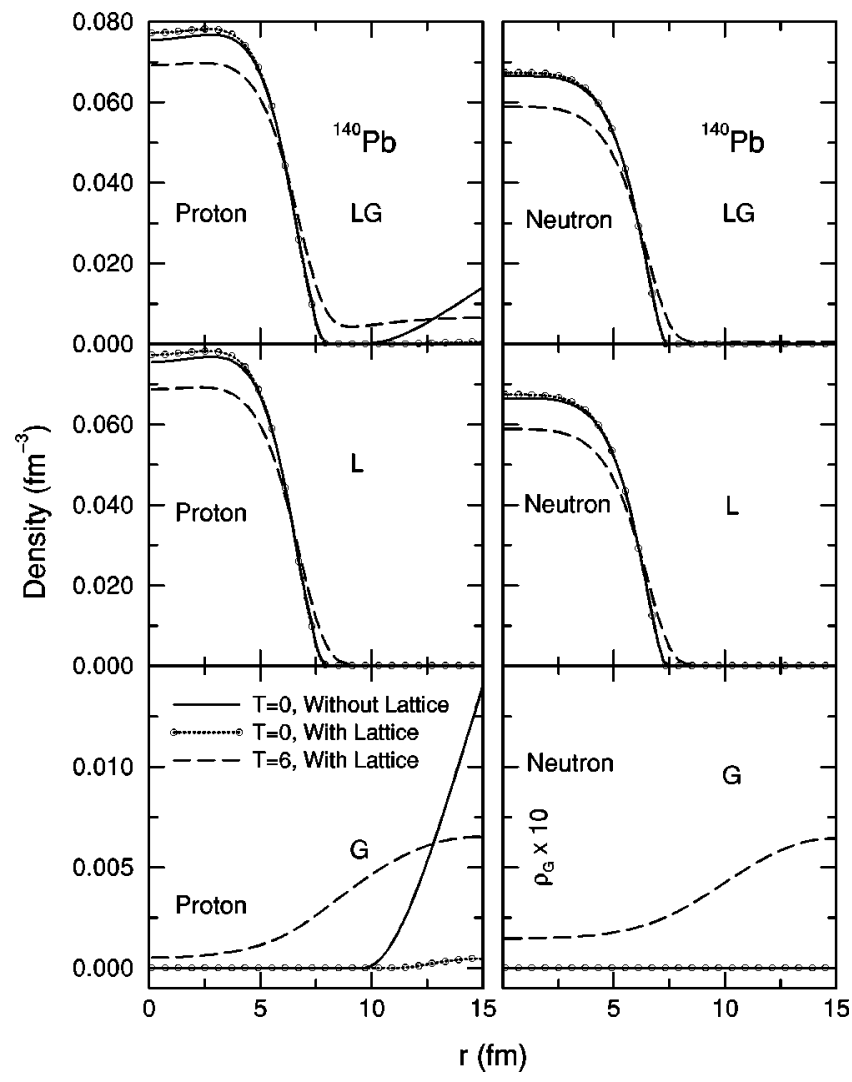

FIG. 4. Same as Fig. 3 but for ${ }^{140} \mathrm{~Pb}$. Here the left panel corresponds to protons and the right panel refers to neutrons.

of Fig. 5 (neutrons and protons), one observes the growth of the neutron or proton skins with positive or negative asymmetry, the effect being more noticeable at zero than at finite temperature.

We now explore the ambient conditions in which a particular nucleus can exist at a certain temperature in the inner crust of the neutron star. For this purpose, we have chosen ${ }^{80} \mathrm{Ca}$ and ${ }^{170} \mathrm{Sn}$ as representative systems. The nucleus ${ }^{80} \mathrm{Ca}$ is well beyond the Thomas-Fermi drip line $\left({ }^{68} \mathrm{Ca}\right.$ being on the drip line) whereas ${ }^{170} \mathrm{Sn}$ is just at the drip boundary. As mentioned in Sec. II, among the four variables $\langle\rho\rangle, Y_{p}, Y_{\nu}$, and $R_{c}$, only one is independent once the $N_{c l}$ and $Z_{c l}$ values of the nucleus are constrained and $\beta$ equilibrium assumed. For the existence of the particular isotope the values of the rest of the variables are then fixed. The correlations among $\langle\rho\rangle, Y_{p}$, and $R_{c}$ are displayed in Fig. 6 for the aforementioned two isotopes at $T=0,3$, and $6 \mathrm{MeV}$. The filled circles correspond to different values of $R_{c}$ at which the calculations have been performed. The values of $R_{c}$ range from $12 \mathrm{fm}$ to $30 \mathrm{fm}$ at a step of $3 \mathrm{fm}$, increasing with decreasing $\langle\rho\rangle$ for all the cases shown. The nucleus ${ }^{80} \mathrm{Ca}$ is beyond the neutron drip line, it is hence embedded in a neutron sea even at zero temperature. For the isolated nucleus, the density of the neutron gas is independent of the box size. Even in calculations with inclusion of effects from lattice electrons, the neutron chemical potential $\mu_{n}$ and hence $\rho_{G}^{n}$ change very little with the cell size. This explains the gradual fall of $\langle\rho\rangle$ and $Y_{p}$ with increasing $R_{c}$. For ${ }^{170} \mathrm{Sn}$, since the nucleus is at the

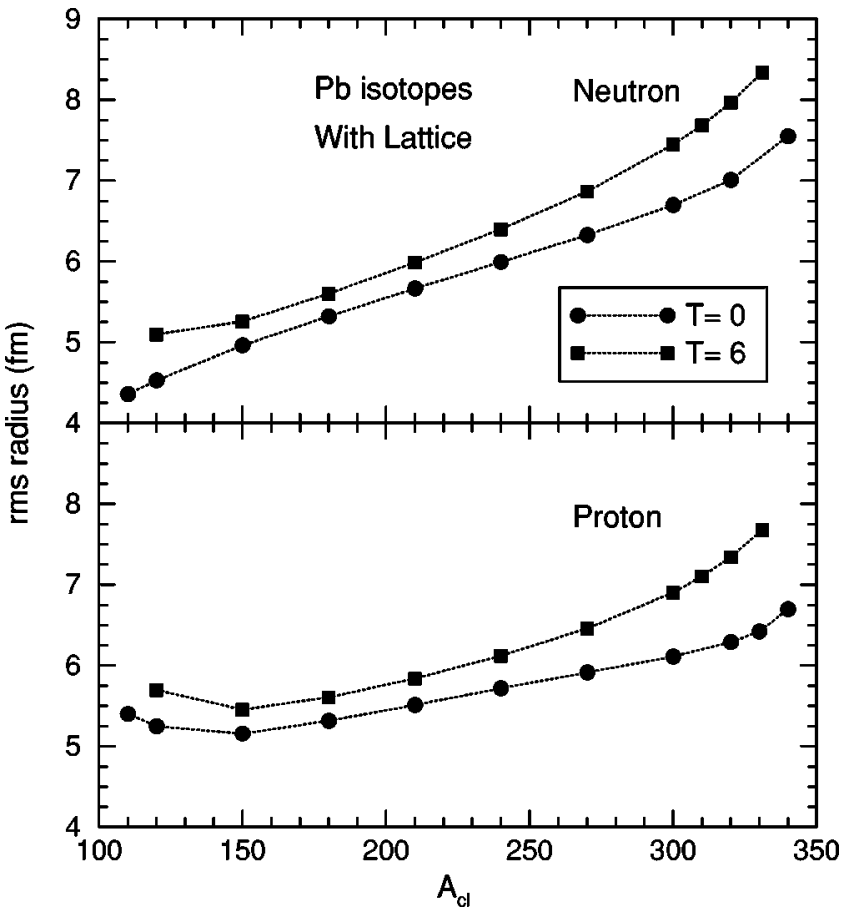

FIG. 5. The neutron (top panel) and proton (bottom panel) rms radii at $T=0$ and $6 \mathrm{MeV}$ calculated in a lattice cell of size $R_{c}$ $=15 \mathrm{fm}$ for $\mathrm{Pb}$ isotopes.

neutron drip boundary, the fall of $\langle\rho\rangle$ with increasing $R_{c}$ is vertical at $T=0$ as there is no neutron gas in the cell. At finite temperature, however, the cluster becomes embedded in a nucleonic gas of mostly neutrons and some protons causing a reduction in $\langle\rho\rangle$ and $Y_{p}$ with increasing $R_{c}$.

The ambient conditions for stability are investigated further for $\mathrm{Pb}$ isotopes ranging from $A_{c l}=110$ to $A_{c l}=330$ at the same three temperatures mentioned for ${ }^{80} \mathrm{Ca}$ and ${ }^{170} \mathrm{Sn}$. The calculations for $\mathrm{Pb}$ have been performed at the value of cell size $R_{c}=15 \mathrm{fm}$. The results are displayed in Fig. 7. The proton drip point for lead at $T=0$ is at $A_{c l}=143$, while the neutron drip point is at $A_{c l}=276$. These points are indicated by the vertical arrows in the figure. In the upper panel of this figure, the average density $\langle\rho\rangle$ is plotted against mass number $A_{c l}$. The curve for the zero temperature case has a minimum at $A_{c l} \sim 143$, which is the proton drip point. Between the drip points ( $A_{c l}=143$ to 276 ), since there is no nucleonic gas around at $T=0$, the average density $\langle\rho\rangle$ within the WS cell increases linearly with mass number. Beyond the drip points, the nuclei are surrounded by a nucleonic gas for their stability, $\langle\rho\rangle$ therefore increases faster. For a hot nucleus there are evaporated nucleons in the cell and the average density is larger.

The proton fraction $Y_{p}$ as a function of the mass number is shown in the middle panel of Fig. 7. A kink is seen to occur at $A_{c l}=143$, the proton drip point below which $Y_{p}$ rises faster because of the presence of the proton gas. For $A_{c l}>143$, the charge $Z_{c l}$ is fixed. Hence, with increase of mass number, $Y_{p}$ decreases linearly up to the neutron drip point beyond which the slope of the curve changes as neutrons appear in the drip phase. With temperature, because of evaporation of nucleons taking place, the kinks smoothen 


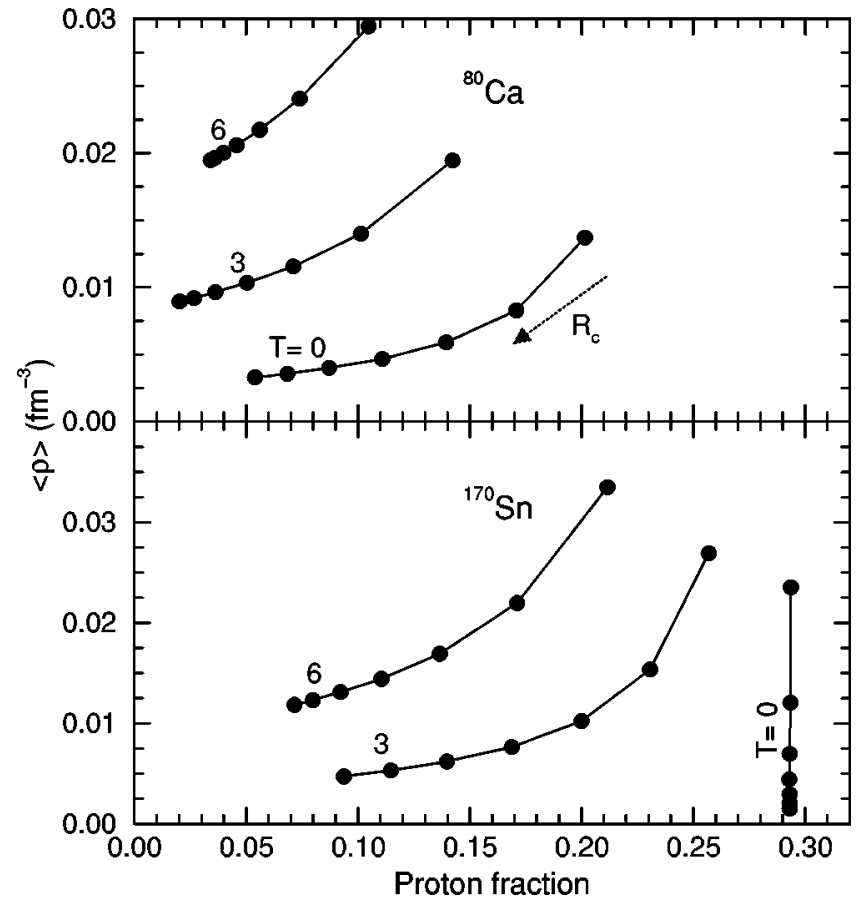

FIG. 6. The average density and proton fraction of the nuclear matter in the Wigner-Seitz cell calculated for the nuclear clusters ${ }^{80} \mathrm{Ca}$ and ${ }^{170} \mathrm{Sn}$. The size $R_{c}$ of the cell is varied from $12 \mathrm{fm}$ to 30 $\mathrm{fm}$ at a step of $3 \mathrm{fm}$, as marked by the filled circles. The calculations are done for temperatures $T=0,3$, and $6 \mathrm{MeV}$. The full lines are drawn to guide the eye.

out, but the overall qualitative features are the same. The lower values of $Y_{p}$ at finite temperature reflect the predominance of neutrons in the gas for isotopes that are not very proton rich. The variation of the neutrino fraction $Y_{\nu}$ $\left(=\rho_{\nu} /\langle\rho\rangle\right)$ with the isotope mass is displayed in the bottom panel. With increasing mass number, it is found to decrease. The effect of temperature on $Y_{\nu}$ is very weak. Inspection of Fig. 7 reveals that for a particular $\langle\rho\rangle, A_{c l}$ is generally double valued. If the isotope is proton rich, $Y_{p}$ is necessarily large and $Y_{\nu}$ also comes out to be large. For the same value of $\langle\rho\rangle$, the neutron-rich isotope is formed when $Y_{\nu}$ is small.

So far our calculations have been done by fixing a given cluster $\left(N_{c l}, Z_{c l}\right)$, which yields various sets of values for the parameters of the problem. A common practice is, however, to fix the parameters such as $\langle\rho\rangle$ and $Y_{p}$ and study the change in the internal structure of neutron star matter. When $\beta$ equilibrium is assumed, for a given value of $\langle\rho\rangle$ and $Y_{p}$, the free energy density (equivalent to free energy per baryon in the present case) in the system of nucleons, electrons, and neutrinos is a minimum in any given cell size $R_{c}$. The cell size sets the periodicity in the neutron star matter; it is not $a$ priori known and therefore is taken as a parameter. Even for given values of $\langle\rho\rangle$ and $Y_{p}$, the neutrino concentration $Y_{\nu}$ (under $\beta$ equilibrium conditions) would be different for different values of $R_{c}$. One may look for a global minimum in the free energy density by varying $Y_{\nu}$ (through variation of $R_{c}$ ). This is displayed in Fig. 8 for $T=0$ and for three values of densities keeping the proton concentration $Y_{p}$ fixed at 0.3 . The minima in the free energy density are marked by arrows.

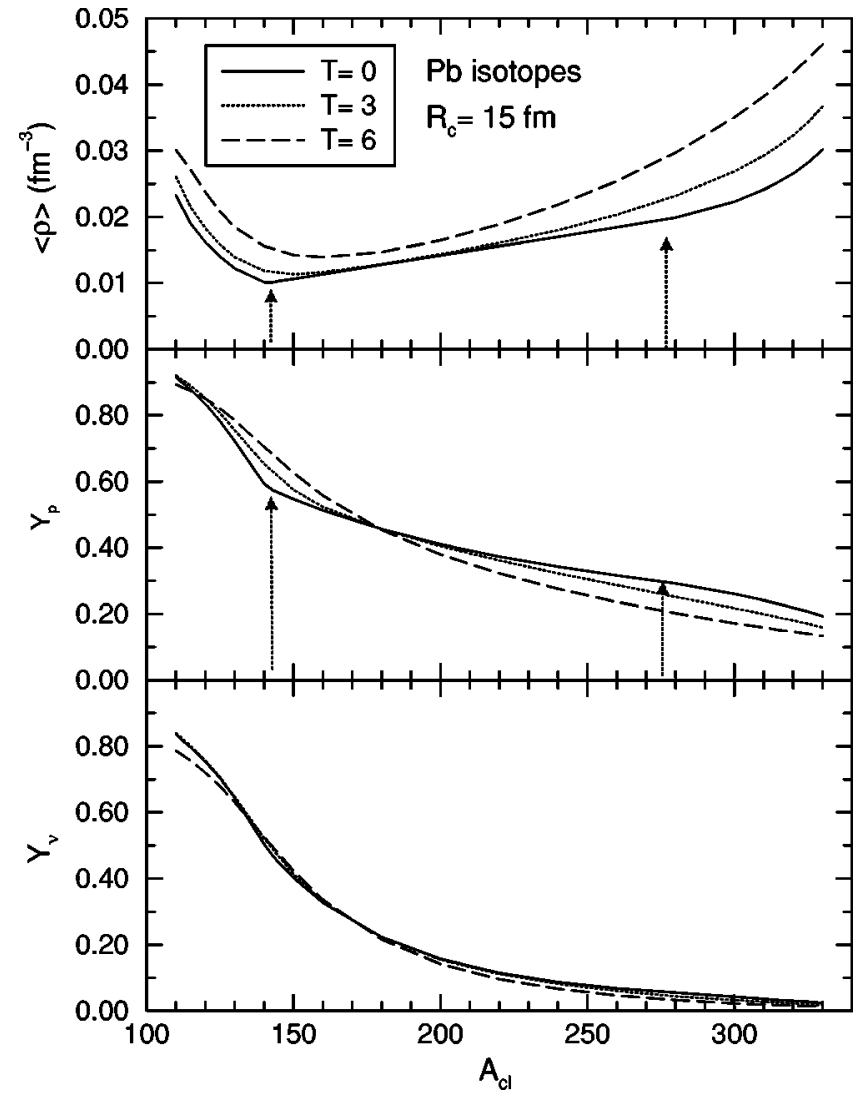

FIG. 7. The variation of the average density $\langle\rho\rangle$, the proton fraction $Y_{p}$, and the neutrino fraction $Y_{\nu}$ as a function of the mass number $A_{c l}$ of the $\mathrm{Pb}$ isotopes calculated in a Wigner-Seitz cell of size $15 \mathrm{fm}$. The calculations are done at $T=0,3$, and $6 \mathrm{MeV}$. The vertical arrows indicate the proton and neutron drip points.

With decreasing density, the cell size corresponding to the global minimum increases.

To understand the origin of the minimum in the free energy profile presented in Fig. 8, the variation of its different components with lattice size for two values of $\langle\rho\rangle=0.001$ (left panel) and $0.01 \mathrm{fm}^{-3}$ (right panel) are shown in Fig. 9. Here $Y_{p}$ is fixed at 0.3. Since these calculations are carried out at zero temperature, the total energy per baryon $e_{t o t}$ $=F / A$ is given by

$$
F / A=e_{N}+e_{l a t}+e_{e}+e_{\nu} .
$$

The different contributions $e_{N}, e_{l a t}, e_{e}$, and $e_{\nu}$ are the nuclear energy including the Coulomb interaction among the protons, the lattice energy (i.e., the Coulomb interaction energy due to the presence of the electrons), the electron kinetic energy, and the neutrino energy per baryon, respectively. It is seen that $e_{N}$ has a minimum at a lattice radius $R_{c}$ somewhat smaller compared to $R_{c}^{\min }$, the value of $R_{c}$ at which $F / A$ is minimum in Fig. 8 at the corresponding $\langle\rho\rangle$. This may be understood from the variation of the cluster size $A_{c l}$ with $R_{c}$ as shown in the middle panels of the figure. The cluster size increases monotonically with $R_{c}$ and for these neutron-rich clusters the minimum binding energy per nucleon occurs at around $A_{c l} \sim 100$ (for nuclei on the 


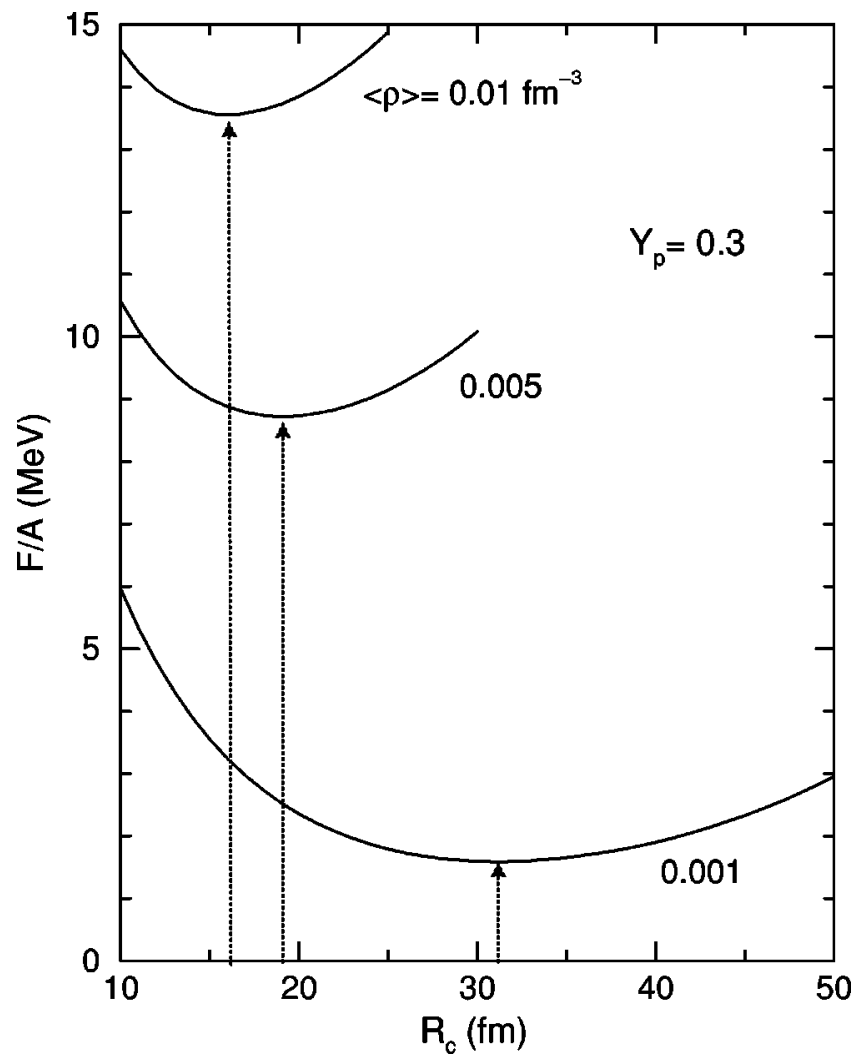

FIG. 8. The variation of the free energy per baryon $F / A$ with the cell size at average densities $\langle\rho\rangle=0.001,0.005$, and $0.01 \mathrm{fm}^{-3}$, the proton fraction being fixed at $Y_{p}=0.3$. The calculations correspond to $T=0$. The vertical arrows refer to the minima in $F / A$.

$\beta$-stability line, this occurs for ${ }^{56} \mathrm{Fe}$ ). The lattice energy $e_{\text {lat }}$ decreases monotonically with $R_{c}$. This is because for given $Y_{p}$, the proton number and hence the electron number increases with increasing lattice size keeping the electron density constant, as a result of which $e_{\text {lat }} \sim-A^{2 / 3}[5]$. The electron kinetic energy per baryon $e_{e}$ is a constant for a given density and $Y_{p}$ and therefore is not shown in the figure. The neutrino energy $e_{\nu}$ passes through a minimum at a value of $R_{c}$ somewhat smaller than $R_{c}^{\min }$ where the neutrino fraction $Y_{\nu}$ is also a minimum (shown in the bottom panel). The competition between $e_{l a t}$ and the rising parts of $\left(e_{N}+e_{\nu}\right)$ determines the location of the minimum in the total free energy at $T=0$.

In Fig. 10, we display the thermal evolution of the cluster composition $\left(A_{c l}, Z_{c l}\right)$ at different fixed densities, at a particular value of $Y_{p}=0.3$ and for a lattice size $R_{c}=15 \mathrm{fm}$. The number of neutrons and protons in the WS cell is then fixed. The full lines correspond to the mass number $A_{c l}$ of the cluster and the dotted lines refer to its charge. For the chosen conditions, the clusters formed at zero temperature in the WS cell comprise all the nucleons without any gas. As the temperature rises, the cluster size shrinks and becomes surrounded by the gas of the evaporated nucleons, the nuclear liquid and the gas being in thermodynamic equilibrium. The cluster evaporates completely at a particular temperature depending on the chosen density, and then the cell contains only the gas of nucleons and the leptons. We call it

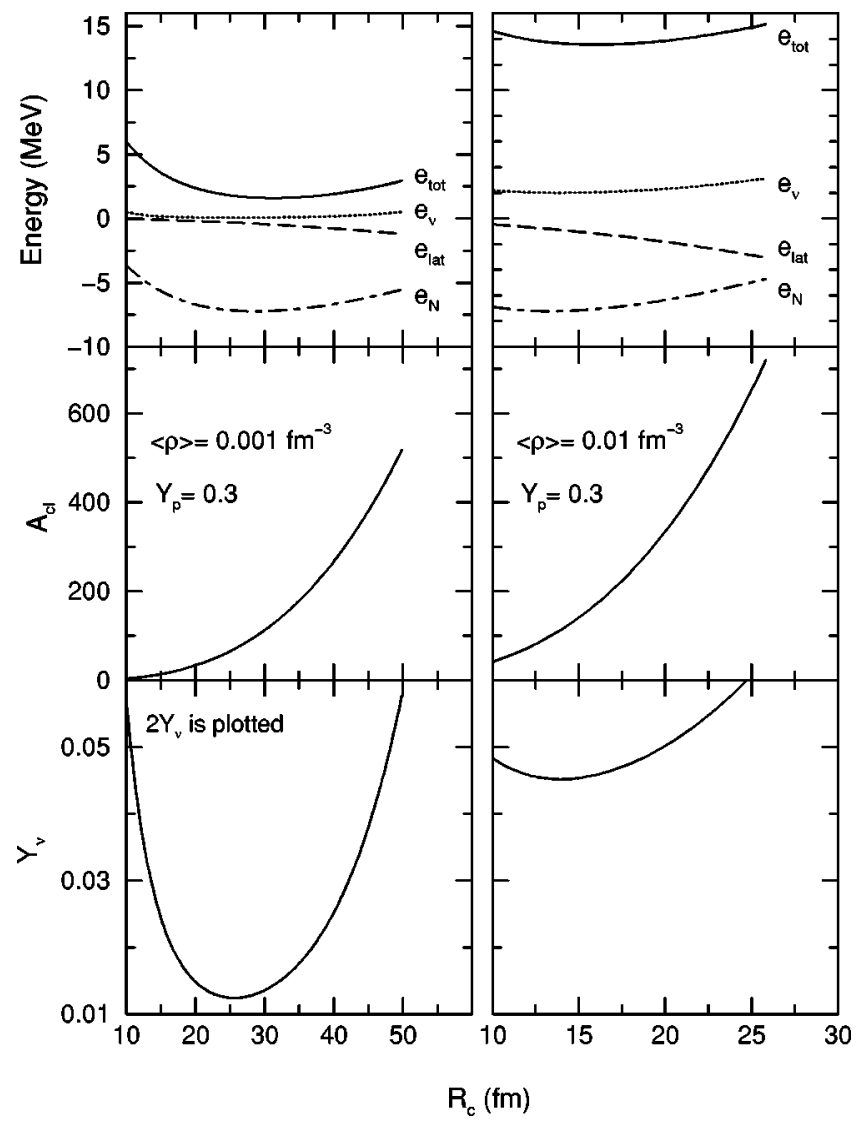

FIG. 9. The different components of the free energy per nucleon as a function of $R_{c}$ are shown in the upper panel for two different values of $\langle\rho\rangle$, at $Y_{p}=0.3$ and $T=0$. The middle panels show the growth of the nuclear cluster with cell size. The bottom panels display the variation of $Y_{\nu}$ as a function of $R_{c}$. The numbers on the left bottom panel are to be scaled down by a factor of two.

the boiling temperature $T_{B}$. As the density $\langle\rho\rangle$ is decreased, the temperature $T_{B}$ falls down. A similar situation was observed in the context of the liquid-gas phase transition in finite nuclei, where the phase transition temperature was found to decrease [26] with an increase in the so-called freeze-out volume or a lowered average density $\langle\rho\rangle$. Figure 10 also tells us about the appearance of different nuclear clusters as the neutron star evolves in the formation stage. Initially, the temperature $T$ may be as high as $\sim 10 \mathrm{MeV}$ and then all the nucleons will likely be in the gas phase. As time flows by, the system cools down and seeds of nuclei with increasing size start appearing from the gas.

\section{CONCLUSIONS}

We have investigated the structural properties of nuclei and nuclear matter as can be found in the environment of the inner crust of a neutron star. These nuclei are dipped in a sea of electrons, neutrinos, and of low-density nucleons. Because of the pressure exerted by the surrounding nucleonic gas, nuclei may exist even far beyond the nominal nuclear drip line. Following the BLV subtraction procedure, we give a prescription in a Thomas-Fermi framework to properly isolate the nucleus from its environment. This is extremely 


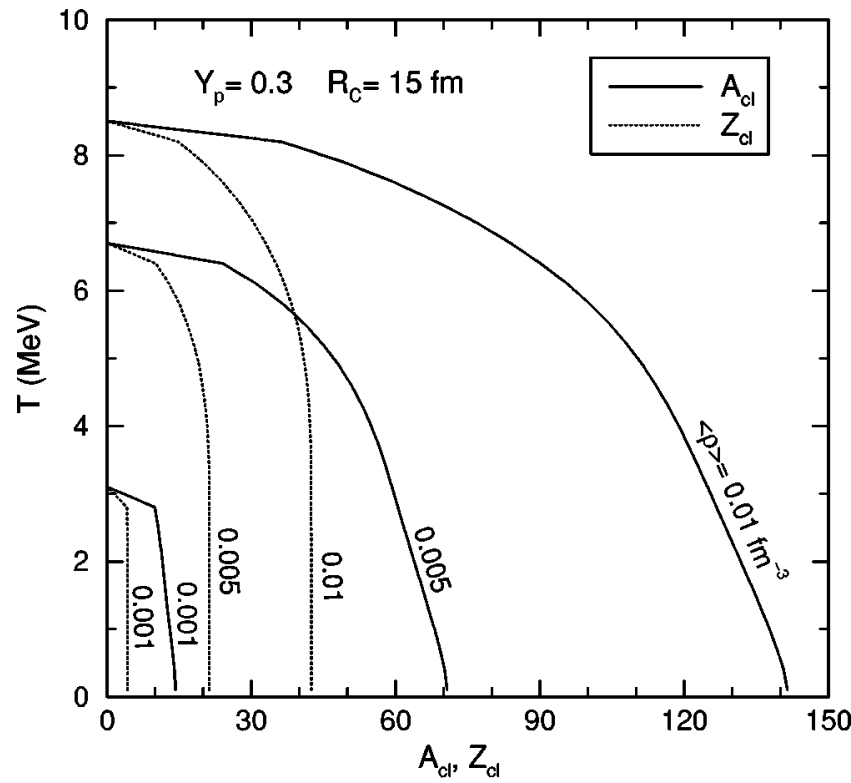

FIG. 10. Thermal evolution of the cluster sizes for average densities $\langle\rho\rangle=0.001,0.005$, and $0.01 \mathrm{fm}^{-3}$. The full lines correspond to the mass number $A_{c l}$ and the dotted lines refer to the atomic number $Z_{c l}$. The calculations are performed with a fixed proton fraction $Y_{p}=0.3$ and a cell size $R_{c}=15 \mathrm{fm}$.

helpful in further exploring the limits of stability.

From our calculations, a limiting asymmetry in the neutron-protron concentration emerges beyond which a nucleus even within the gaseous environment ceases to exist. A delicate balance between the Coulomb force and the di- luted surface tension with increasing asymmetry and increasing density of the environment possibly plays a pivotal role here. We also investigate the ambient conditions, namely, the average density, electron or proton fraction, and the neutrino concentration, under which nuclei of a particular species can be formed at different temperatures in the stellar matter. We, furthermore, study the thermal evolution of the nuclear clusters at different densities, which may serve as a guide to understand the nucleation process of different species of nuclear clusters from the nucleonic sea as the neutron star cools down in the earlier stages of its formation.

We have left our calculations on a simple pedestal. That is, we have not included the extension of the Thomas-Fermi framework, we have not taken the shell effects into account, possible plasma effects have been ignored and, similarly, the presence of $\alpha$ particles at low densities has not been taken into consideration. We have worked with the $\mathrm{SKM}^{*}$ interaction; at large asymmetry, its validity is not unquestionable. The method we have employed for the separation of the liquid and the gas part following the BLV prescription is also not beyond criticism. Sophistications in the approach or the use of more suitable interactions may change the results somewhat, but the qualitative features that emerge from our calculations, we believe, will remain mostly unchanged.

\section{ACKNOWLEDGMENTS}

Two of us (X.V. and M.C.) acknowledge financial support from the DGICYT (Spain) under Grant No. PB98-1247 and from DGR (Catalonia) under Grant No. 2001SGR-00064.
[1] G. Baym, H. A. Bethe, and C. J. Pethick, Nucl. Phys. A175, 225 (1971).

[2] J. R. Buchler and Z. Barkat, Phys. Rev. Lett. 27, 48 (1971).

[3] C. J. Pethick and D. G. Ravenhall, Annu. Rev. Nucl. Part. Sci. 45, 429 (1995).

[4] H. Heiselberg and M. Hjorth-Jensen, Phys. Rep. 328, 237 (2000).

[5] S. L. Shapiro and S. A. Teukolsky, Black Holes, White Dwarfs, and Neutron Stars (Wiley, New York, 1983).

[6] W. D. Langer, L. C. Rosen, J. M. Cohen, and A. G. W. Cameron, Astrophys. Space Sci. 5, 529 (1969).

[7] D. G. Ravenhall, C. J. Pethick, and J. R. Wilson, Phys. Rev. Lett. 50, 2066 (1983).

[8] K. Oyamatsu, Nucl. Phys. A561, 431 (1993).

[9] C. P. Lorenz, D. G. Ravenhall, and C. J. Pethick, Phys. Rev. Lett. 70, 379 (1993).

[10] K. S. Cheng, C. C. Yao, and Z. G. Dai, Phys. Rev. C 55, 2092 (1997).

[11] L. Engvik, E. Osnes, M. Hjorth-Jensen, G. Bao, and E. Østgaard, Astrophys. J. 469, 794 (1996); J. M. Lattimer and M. Prakash, ibid. 550, 426 (2001).

[12] J. M. Lattimer and D. G. Ravenhall, Astrophys. J. 223, 314 (1978).

[13] D. Q. Lamb, J. M. Lattimer, C. J. Pethick, and D. G. Raven- hall, Nucl. Phys. A360, 459 (1981).

[14] H. Müller and B. D. Serot, Phys. Rev. C 52, 2072 (1995).

[15] J. M. Lattimer, C. J. Pethick, D. G. Ravenhall, and D. Q. Lamb, Nucl. Phys. A432, 646 (1985).

[16] W. D. Myers, W. J. Swiatecki, and C. S. Wang, Nucl. Phys. A436, 185 (1985).

[17] M. Centelles, M. Del Estal, and X. Viñas, Nucl. Phys. A635, 193 (1998)

[18] P. Bonche, S. Levit, and D. Vautherin, Nucl. Phys. A427, 278 (1984); A436, 265 (1985).

[19] E. Suraud, Nucl. Phys. A462, 109 (1987).

[20] J. N. De, X. Viñas, S. K. Patra, and M. Centelles, Phys. Rev. C 64, 057306 (2001).

[21] M. Pi, X. Viñas, M. Barranco, A. Perez-Canyellas, and A. Polls, Astron. Astrophys., Suppl. Ser. 64, 439 (1986).

[22] M. Brack, C. Guet, and H. B. Håkansson, Phys. Rep. 123, 275 (1985).

[23] J. P. Cox and R. T. Giuli, Principles of Stellar Structure (Gordon and Breach, New York, 1968).

[24] J. Besprosvany and S. Levit, Phys. Lett. B 217, 1 (1989).

[25] J. N. De, D. Bandopadhyay, S. K. Samaddar, and N. Rudra, Nucl. Phys. A534, 294 (1991).

[26] J. N. De, B. K. Agrawal, and S. K. Samaddar, Phys. Rev. C 59, 1 (1999). 